\title{
Perspective
}

PERSPECTIVE Actualité en histoire de l'art

2| 2007

La Grande-Bretagne/Période moderne

\section{Histoire de l'histoire de l'art en Grande-Bretagne : grandes tendances et nouveaux débats}

\section{Stephen Bann, Nicholas Penny et Patricia Rubin}

\section{(2) OpenEdition}

1 Journals

\section{Édition électronique}

URL : http://journals.openedition.org/perspective/3800

DOI : $10.4000 /$ perspective.3800

ISSN : 2269-7721

Éditeur

Institut national d'histoire de l'art

\section{Édition imprimée}

Date de publication : 30 juin 2007

Pagination : 207-230

ISSN : 1777-7852

\section{Référence électronique}

Stephen Bann, Nicholas Penny et Patricia Rubin, « Histoire de I'histoire de l'art en Grande-Bretagne: grandes tendances et nouveaux débats », Perspective [En ligne], 2 | 2007, mis en ligne le 31 mars 2018, consulté le 01 octobre 2020. URL : http://journals.openedition.org/perspective/3800 ; DOI : https:// doi.org/10.4000/perspective.3800 


\title{
Histoire de l'histoire de l'art en Grande-Bretagne : grandes tendances et nouveaux débats
}

\author{
Stephen Bann, Nicholas Penny et Patricia Rubin
}

\section{Stephen Bann | Histoire de I'histoire de l'art en Grande- Bretagne: grandes tendances et nouveaux débats}

1 Après l'apport des historiens de l'art européens arrivés en Angleterre dans les années 1930, où en est l'histoire de l'art en Grande-Bretagne? Pour comprendre l'évolution récente de l'historiographie britannique (ou anglo-saxonne?), s'est rapidement imposé l'intérêt de traduire l'article de Stephen Bann ("The History of Art History in Britain: a Critical Context for Recent Developments and Debates ») extrait du recueil édité par Peter Burke History and Historians in the Twentieth Century publié par la British Academy en 2002. [Perspective]

2 Il est bien délicat de rendre compte de l'évolution de l'histoire de l'art en Grande Bretagne $\mathrm{au} \mathrm{xx}^{\mathrm{e}}$ siècle, pour des raisons qui relèvent de l'histoire de la discipline dans son ensemble mais qui semblent aussi particulièrement pertinentes dans le contexte britannique. Au début du siècle, l'histoire de l'art en tant que discipline universitaire n'avait pratiquement aucune existence institutionnelle en Grande-Bretagne, bien que et c'est le point qui sera discuté ici - la tradition de critique esthétique ait fourni une base différente pour la compréhension et l'évaluation des arts. À la fin du siècle, ce vide était remplacé par un foisonnement. L'histoire de l'art, à la suite de la littérature anglaise, et avant les études cinématographiques, était venue s'insérer, de façon bien visible et populaire, dans le panorama des sciences humaines.

3 Il reste cependant la question de savoir si le concept d'une histoire de l'art « nationale » a un sens. Depuis la fin de la Seconde Guerre mondiale, tous les domaines de la 
recherche universitaire ont plus ou moins pris une dimension internationale; en ce qui concerne la toute nouvelle discipline de l'histoire de l'art, le processus a même été particulièrement spectaculaire. Cela parce que, d'une part, le monde anglophone a été essentiellement nourri par l'histoire de l'art allemande et qu'il a donc été en contact avec la tradition originaire, et la plus riche, d'écrits savants dans le domaine ${ }^{1}$, et que, d'autre part, la discipline a bénéficié des échanges très fertiles entre la GrandeBretagne et les États-Unis pendant les décennies suivantes. Une grande partie des chercheurs vivants qui seront mentionnés ici a occupé des postes des deux côtés de l'Atlantique ${ }^{2}$, ce qui rend d'autant plus difficile d'isoler une lignée typiquement britannique, bien que les différences par rapport aux pays du continent soient toujours perceptibles. Dans ce court essai, je ne vais pas entreprendre un examen approfondi de l'existence d'une tradition d'écriture nationale complexe sur les arts visuels - tradition qui, dans les premières années du siècle, divergeait sensiblement de l'historicisme de la tradition germanique. Je ne vais pas non plus examiner en détail l'histoire passionnante de la réception et de l'appropriation nationale de l'histoire de l'art allemande en Grande-Bretagne. Ces aspects ne seront qu'effleurés. Je porterai plus particulièrement mon attention sur les questions les plus récemment débattues au sein d'une communauté de spécialistes «anglo-saxons» au sens large. Toutefois, ces débats trouvent clairement leur origine dans les conditions spécifiques de l'évolution de l'histoire de l'art tout au long du siècle.

\section{L'écriture britannique sur l'art et l'histoire de l'art germanique}

4 La discipline de l'histoire de l'art possède l'une des plus anciennes associations du monde, le Comité international d'histoire de l'art (CIHA). Fondé en 1873, il contredit partiellement son appellation dans la mesure où sa base première était l'Europe centrale. Son quatrième congrès international, tenu à Budapest en 1896, fut le premier à accueillir des participants français. Lorsque le quinzième congrès eut lieu à l'University College de Londres en 1939, l'histoire de l'art britannique avait peu ou prou atteint sa maturité. Le Courtauld Institute, fondé à Londres en 1931, était en passe de devenir le premier établissement consacré à l'enseignement et à la recherche sur le sujet. Dans le numéro du quotidien The Times qui couvrait le congrès du CIHA, une colonne annonçait en parallèle la fondation du Barber Institute à l'Université de Birmingham ${ }^{3}$. À la même époque, un noyau d'historiens d'art allemands émigrés, dont Edgar Wind et Fritz Saxl, s'était déjà formé autour de la bibliothèque du chercheur indépendant Aby Warburg, qui avait quitté Hambourg, sa ville natale, pour Londres. Peu de temps après la fin de la guerre, cela allait aboutir à la création du Warburg Institute, centre de recherche relevant de l'Université de Londres.

5 Après avoir mentionné ces débuts prometteurs, il serait cependant légitime de glisser une note en guise d'avertissement. Au début des années 1930, le premier magazine d'art britannique, créé en 1903 sous le titre The Burlington Magazine for Connoisseurs, avait déjà trois décennies d'existence. D'abord sous la direction d'Herbert Read, puis de Benedict Nicolson, il allait accueillir parmi ses premiers collaborateurs des spécialistes comme Kenneth Clark et Ellis Waterhouse, ainsi que Roger Fry, qui s'y distinguera. Ces noms ne font toutefois que souligner le fait que, tout au long de l'entre-deux-guerres, l'histoire de l'art en Grande-Bretagne était restée en grande partie la chasse gardée des grands musées dont les conservateurs et trustees étaient membres aussi bien du comité de rédaction du Burlington que du comité d'honneur de la conférence du CIHA en 1939. 
Si les prestigieux musées des universités britanniques, comme l'Ashmolean à Oxford et le Fitzwilliam à Cambridge, avaient continué d'enrichir leurs collections dans la première partie du $\mathrm{xx}^{\mathrm{e}}$ siècle, on ne trouvait aucune trace d'une évolution parallèle de l'histoire de l'art en tant que discipline universitaire. Ainsi à Cambridge, la première chaire d'histoire de l'art est créée dans les années 1960 au sein d'une nouvelle faculté, en association avec l'architecture. À Oxford, la chaire Slade Professor ${ }^{4}$ fut offerte à Edgard Wind lorsque ce dernier décida de ne pas rejoindre ses collègues du Warburg après la guerre, mais l'histoire de l'art allait rester fermement maintenue sous la tutelle de la faculté d'histoire.

6 Signaler cette disparité entre l'histoire de l'art sur le continent et l'histoire de l'art en Grande-Bretagne serait vain s'il n'y avait l'intention de présenter la situation sous un jour plus favorable. Dans la Grande-Bretagne du XIX ${ }^{e}$ siècle, la culture artistique s'était précisément développée sur l'exemple de figures qui avaient comblé le fossé entre la pratique et la théorie, le musée et l'académie: le redoutable Sir Charles Eastlake, président de la Royal Academy ainsi que premier directeur de la National Gallery, en était un exemple frappant. C'est en partie à son œil clairvoyant que la collection de ce musée doit son exceptionnelle richesse ${ }^{5}$. En même temps, au cours du siècle, les conditions particulières de la culture britannique avaient donné naissance à une tradition d'écriture spécifique aux arts visuels, dont l'appellation de «critique esthétique " revendique précisément la nature littéraire, mais qui mérite une reconnaissance dans le domaine de l'art, ne serait-ce que pour le prestige et l'influence qu'elle a rapidement acquis dans toute l'Europe ainsi qu'en Amérique du Nord. John Ruskin est le représentant le plus notoire de cette tradition, mais ses écrits, nourris d'un fort moralisme et d'une attention à l'actualité, sont forcément en contradiction avec les valeurs de l'Histoire. Walter Pater, quant à lui, reflète au mieux à quel point cette tradition a pu être appliquée à la critique d'une histoire de l'art positiviste. Son rejet, poli mais ferme, du «nouveau Vasari » - l'étude de Joseph Archer Crowe et Giovanni Battista Cavalcaselle sur l'histoire de la peinture italienne - ne revêt pas tant une tolérance à l'égard des positions anhistoriques qu'il ne manifeste le désir de prendre en compte plus largement les contextes culturels quand il s'agit d'évaluer une influence stylistique. En ce sens, W. Pater peut véritablement être considéré comme ayant anticipé le concept d'une "culture visuelle " conçue comme une alternative à l'étroitesse d'un connoisseurship fondé sur l'étude de l'objet ${ }^{6}$.

7 Évidemment, la critique esthétique n'existait pas (et n'existe toujours pas) totalement en opposition à l'histoire de l'art. Dans les dernières années du XIX siècle, John Addington Symonds fut l'un des principaux écrivains à privilégier un mode d'écriture historique et narrative plutôt qu'essayiste, dans ses brillantes et influentes études sur l'art de la Renaissance italienne, mode qui culmina dans son dernier ouvrage important, en trois volumes: The Life of Michelangelo Buonarotti (1893) ${ }^{7}$. Cependant, il serait juste de dire que, parmi les contributions les plus importantes à l'histoire de l'art par des auteurs britanniques, un bon nombre continua de refléter, très avant dans le $\mathrm{xx}^{\mathrm{e}}$ siècle, l'ambivalence inhérente à la forme de l'essai - ce qui implique un lien avec la critique d'art - de préférence à une écriture selon le mode positiviste, et encore moins une étude fondée sur la philosophie et la théorie. Cela s'applique assurément à Roger Fry, dont la prééminence comme critique d'art contemporain structure toute l'approche, qu'il écrive sur Giotto ou sur Cézanne. Cela s'applique également à l'écrivain qui, plus qu'aucun autre, reprit le flambeau de la critique esthétique du xIX 
siècle, Adrian Stokes. En effet, la fortune inégale des écrits de ce dernier, depuis sa mort en 1972, reflète précisément la difficulté de placer un personnage aussi considérable et aussi complexe dans le paradigme des écrits sur l'histoire de l'art, de plus en plus dominés par le précédent des théoriciens germaniques ${ }^{8}$.

Sur le long terme, il semble qu'un bon nombre de valeurs constantes de la tradition de l'écriture britannique sur l'art soient redécouvertes par certains de ses praticiens actuels les plus éminents. L'une des études les plus originales et les plus admirées des deux dernières décennies, Painting as an Art (1987), est l'œuvre d'un ami et disciple d'Adrian Stokes, Richard Wollheim, qui a appliqué le même type d'intuition à une série de tableaux importants, tout en respectant leur contexte historique. Même lorsqu'une parenté manifeste est décelée entre les méthodes des historiens d'art allemands et celles de leurs suiveurs britanniques, cela ne doit pas détourner l'attention des autres qualités propres à ces derniers. Par exemple, l'influence manifeste que l'approche marxiste de Frederick Antal a exercée sur Anthony Blunt ne doit pas masquer la vision du monde, très différente, qui ressort dans les écrits de ce dernier sur Poussin. De même que la reconnaissance de la dette de Michael Baxandall envers Ernst H. Gombrich ne doit pas détourner l'attention de la direction totalement nouvelle dans laquelle ses subtiles analyses sur la dimension matérielle de la peinture ont été conduites 9

Pour prendre un autre exemple frappant, le décès de Francis Haskell, en 2000, fournit une occasion, triste mais éclairante, de revenir sur une carrière qui a adopté un modèle très différent de celui des mentors allemands de la génération précédente. Son travail recèle de nombreuses résonances de la tradition britannique du xix siècle : non tant le rythme musical des textes de critique esthétique mais celui des essais percutants et solidement argumentés de leur prédécesseur, William Hazlitt, qui fut l'un des tout premiers auteurs à communiquer la fascination exercée par l'exposition de l'art, dans le contexte spécifique du musée et de la collection ${ }^{10}$. Alors que son prédécesseur à la chaire d'histoire de l'art à Oxford, Edgar Wind, cherchait à éclairer l'iconographie complexe des chefs-d'œuvre de la Renaissance, F. Haskell portait une grande attention aux subtilités du mécénat et aux changements de goût continuels auxquels de tels chefs-d'œuvre avaient eux-mêmes été confrontés au fil des ans. Dans son magistral ouvrage, History and its Images [1993 ; trad. fr. : L'historien et les images, Paris, 1995] ; c'est la nature insaisissable et le manque de fiabilité de l'image en tant que véhicule de la vérité historique qui sont devenus le sujet principal du débat. F. Haskell posait la question de savoir si l'image avait jamais été, et pourrait être un jour, une source fiable pour l'historien. Mais ce faisant, il ouvrait les yeux de ses collègues sur le statut problématique d'une représentation historique. Vu sous cet angle, l'héritage qu'il a transmis revêt une double importance : il signale le tournant historiographique adopté par l'histoire de l'art dans le dernier quart du siècle, et il ouvre également de plus vastes perspectives d'engagement dans l'histoire de la culture visuelle.

\section{Le tournant historiographique}

10 Ce qui précède ne représente que quelques exemples de la façon dont la tradition d'écriture propre à la Grande-Bretagne peut être repérée, parfois sous la forme d'un courant souterrain, dans l'œuvre des historiens d'art contemporains. Cependant, il reste vrai que le facteur capital du demi-siècle suivant la Seconde Guerre mondiale fut l'opportunité, et la nécessité, de rattraper le retard dans l'exigence intellectuelle, par 
rapport à l'approche qui s'était développée dans les pays germanophones. Les points de rencontre entre les historiens d'art allemands immigrés et les chercheurs qui devaient beaucoup à leur enseignement, en Grande-Bretagne comme aux États-Unis, constituent effectivement un sujet complexe qui ne peut être étudié en détail ici. Vu depuis la fin $\mathrm{du} \mathrm{Xx}^{\mathrm{e}}$ siècle, il apparaît qu'une étape supplémentaire a été atteinte dans le processus de réception lorsque le monde anglophone a enfin eu l'opportunité d'avoir accès au registre complet des sources attestées par les mentors allemands. C'est ce que j'appelle le tournant historiographique. Les ouvrages publiés en anglais dans le précédent quart de siècle par des figures aussi importantes qu'Erwin Panofsky, E. H. Gombrich ou E. Wind se référaient implicitement à des débats antérieurs qu'eux-mêmes et leurs prédécesseurs considéraient comme allant de soi. En conséquence, un grand nombre de nouvelles exégèses ont dû être entreprises pour que les études majeures des pionniers allemands soient remises en circulation.

11 Le premier travail important de cette tendance est l'étude influente de Michael Podro, The Critical Historians of Art [1982 ; trad. fr. : Les historiens d'art au XIX siècle, Paris, 1990], qui reconnaissait dans sa préface que la grande tradition de pensée et d'écriture germanique sur les images " continuait d'exercer une influence, le plus souvent sous forme de réminiscences discrètes, les textes proprement dits étant comme éclipsés " ${ }^{11}$. De façon similaire, le Panofsky and The Foundations of Art History de Michael Ann Holly, publié deux ans plus tard, aspirait à redessiner le profil intellectuel complet du personnage, unanimement considéré comme ayant joué un rôle essentiel dans le développement de la discipline, ainsi qu'à évaluer sa contribution par rapport au contexte contemporain ${ }^{12}$.

12 Au cours des années 1990, des ouvrages d'introduction comme ceux-là, qui fournissent une grille de lecture pour la compréhension des méthodes de l'historien d'art, ont été complétés par une nouvelle sélection de textes importants traduits et publiés en anglais. Toute liste, même la plus brève, inclurait Problems of Style d'Alois Riegl, paru en anglais en 1992 [trad. fr.: Questions de style : fondements d'une histoire de l'ornementation, Paris, 1992] avec cette remarque d'Henri Zerner dans sa préface : « [Ce livre] n'a jamais été ni remplacé ni dépassé pour ce qui est de la présentation de l'histoire de l'ornement de l'époque antique et médiévale ${ }^{13}$; Perspective as Symbolic Form d'E. Panofsky, enfin rendu accessible grâce à la traduction anglaise de Christopher Wood qui arrivait à point nommé [1991; trad. fr. : La Perspective comme forme symbolique et autres essais, Paris, 1975], et qui avait acquis "une réputation bien au-delà du territoire spécialisé de l'histoire de l'art ", selon les termes du traducteur dans son introduction ${ }^{14}$; enfin, plus récemment, une étude telle que The Practice of Art History du méconnu Otto Pächt [1999; trad. fr. : Questions de méthode en histoire de l'art, Paris, 1994], avec là encore une préface de C. Wood qui commente "l'ingénuité et la créativité de son regard» et la force spécifique de ses méthodes d'analyse, jugées comparativement aux approches de contemporains plus connus comme E. Panofsky et E. H. Gombrich ${ }^{15}$.

D'un point de vue anglophone, en tout cas, cette accumulation progressive des premiers textes fondamentaux pouvait sembler constituer la formation tardive d'un canon. D'un point de vue extérieur à la discipline, on pouvait estimer que les instruments critiques historiques indispensables étaient déjà présents (même s'ils étaient temporairement inaccessibles) et que, par conséquent, il suffisait de revoir judicieusement l'ensemble du domaine pour rééquilibrer le récit, en corrigeant les irrégularités et les déformations qui auraient pu s'y glisser accidentellement. 
Conformément à cette reprise historiographique, et se développant logiquement à partir d'elle, on vit apparaître une série d'ouvrages synthétiques utilitaires, généralement sous la forme de recueils d'essais, qui cherchaient à appliquer les bénéfices méthodologiques de cette vision élargie aux tâches concrètes des études historiques en art. J'en cite trois en particulier, en notant que leurs titres signalent cette ambition de synthèse: Essential Art History (1992), Critical Terms for Art History (1996), et The Subjects of Art History (1998) ${ }^{16}$.

14 Il est trop tôt pour juger de l'utilité à long terme de tels recueils. Ils sont de toute évidence le produit de ce que les éditeurs universitaires pensaient être le support nécessaire pour des étudiants plus nombreux et de provenances plus diverses et il faut souligner que ces ouvrages avaient été conçus pour combler les lacunes méthodologiques constatées. Voilà qui serait une preuve suffisante pour avancer que l'histoire de l'art a subi un rite de passage lors des deux dernières décennies du $\mathrm{xx}^{\mathrm{e}}$ siècle. En même temps, cela impliquait une plus grande reconnaissance des «fondations" posées par les générations successives de chercheurs de tradition germanique, ainsi que la tentative d'appliquer systématiquement leurs approches à une gamme étendue d'objets divers.

Bien que je présente ce phénomène comme la lecture immédiate d'un bilan de fin de millénaire, je me dois d'ajouter qu'il est loin d'en faire l'histoire complète. Toutes ces indications sur la récente mise au point de la discipline en termes intrinsèques, pour ainsi dire, ne doivent pas conduire à négliger sa situation par rapport aux autres domaines et aux champs d'études adjacents. Il semble que l'histoire de l'art ait gagné le droit de se considérer comme une discipline autonome. Cette revendication est justifiée en ce qui concerne les aspects pédagogiques et institutionnel de la discipline, même si de grandes disparités géographiques semblent perdurer dans l'enseignement et la recherche en l'art, lorsque nous regardons ce qui se passe dans quelques-uns des pays les plus concernés ${ }^{17}$. Mais les disciplines ne peuvent se contenter de lutter pour leur autonomie intellectuelle. Si l'histoire de l'art souffre certainement d'être profondément engagée dans une transformation culturelle trop importante pour qu'elle puisse la contrôler, c'est aussi une chance pour elle. Cette transformation demeure cependant trop intégralement enracinée dans l'identité conflictuelle de la discipline pour être simplement rejetée ou dédaignée.

\section{Le tournant vers le visuel [the pictorial turn] dans les sciences humaines}

Mon dernier point se référait au récent «tournant historiographique » de l'histoire de l'art. Le chercheur américain W.J. Thomas Mitchell a écrit, par contraste, sur le « tournant vers le visuel » à l'œuvre dans toutes les sciences humaines. Étant lui-même l'exemple notoire d'un spécialiste venu du domaine adjacent des études de la langue anglaise et ayant contribué de façon significative à enrichir les techniques de l'histoire de l'art, W. J. T. Mitchell exprime la difficulté (qui est en même temps une opportunité) qui se présente à l'historien d'art d'une façon particulièrement percutante: "Si le tournant vers le visuel se produit effectivement dans les sciences humaines, l'histoire de l'art pourrait très bien voir sa marginalité théorique se transformer en une position intellectuelle centrale, sous la forme d'un défi : proposer le récit de son principal objet théorique - la représentation visuelle - pour qu'il soit utilisable par d'autres disciplines 
des sciences humaines. S'occuper des chefs-d'œuvre de la peinture occidentale ne sera certainement pas suffisant. Une critique interdisciplinaire étendue sera nécessaire, qui prendra en compte des travaux parallèles comme le long combat dans le domaine des études filmiques pour faire coïncider modèles linguistiques et mise en image, ainsi que pour placer le médium filmique dans le contexte plus large de la culture visuelle $»^{18}$.

Il serait facile d'ergoter sur certains points considérés comme allant de soi dans cet exposé. W. J. T. Mitchell a publié pour la première fois son essai en 1992, et sa remarque sur la "marginalité théorique " de l'histoire de l'art reprend la vision, difficile à accepter, de l'état de la discipline dans le monde anglophone avancée quatre ans auparavant par Norman Bryson, dans son Calligram : Essays in New Art History from France $(1988)^{19}$. Il est peu probable qu'un observateur impartial affirmerait aujourd'hui aussi catégoriquement que les études filmiques s'étaient enfin constituées en discipline agréée et que l'histoire de l'art devait aspirer à ce paradigme. Ce qui n'affecte en rien, cependant, la force critique de ce que W. J. T. Mitchell soutient. En termes plus directs, l'attraction irrésistible exercée par l'« image » dans le champ des sciences humaines et au-delà met l'historien d'art dans l'embarras. D'une part, l'histoire de l'art ne peut espérer rester en dehors des demandes que les spécialistes en histoire et en littérature adressent aux données visuelles, encore moins de celles des étudiants en cinéma, en photographie et autres médiums visuels. D'autre part, si l'histoire de l'art déplace ses paramètres pour avoir une vision plus éclectique de son domaine disciplinaire, ce sera sans doute aux dépens des conceptions les plus éprouvées de son rôle.

J'ai l'intention d'examiner quelques-uns des débats récents à la lumière de ce dilemme. Il semble bien que ces problèmes n'affectent pas si fortement les autres domaines pris en compte dans ce volume ${ }^{20}$. Par exemple, la contribution des historiens britanniques $\mathrm{du}$ siècle dernier à notre connaissance des classes sociales, de la vie citadine ou du Moyen Âge peut toujours être considérée légitimement comme une sous-section des études historiques, quelle que soit la nouveauté ou la spécificité de la méthodologie. Il n'est pas aussi évident que l'histoire de l'art puisse, ou doive, être considérée de cette façon. En effet, on pourrait dire qu'en raison de ses liens nécessaires et inévitables avec les problèmes de représentation et de mise en image, elle est et restera un domaine d'étude soumis à de vives tensions, perpétuellement traversé par de nouveaux débats sur la nature de ses objets et les limites incertaines de sa compétence.

Cela ne concerne pas exclusivement, ni même essentiellement, le conflit entre les tendances "conservatrices » ou "avant-gardistes», comme la citation extraite de l'anthologie de N.Bryson de 1988 pourrait le laisser croire. C'est le problème, endémique, de la résistance que l'image oppose au langage, à tous les degrés auxquels les historiens d'art sont disposés à s'affronter. La récente publication de la traduction d'O. Pächt, à laquelle j'ai déjà fait référence, a choisi la citation suivante pour sa quatrième de couverture: "Mon credo pour l'histoire de l'art est celui-ci: Au commencement était l'œil, pas le mot ». Cela implique bien sûr qu'O. Pächt interroge la dissonance entre des interprétations fondées sur la linguistique d'un côté, et les exigences d'expérience visuelle, supposées primordiales, qu'il détecte dans les travaux de certains pionniers allemands, de l'autre ${ }^{21}$. Je ne peux pas traiter ici des questions importantes qui sont à l'origine de tels débats au sujet de la représentation et de l'image. Elles ont été analysées à de nombreuses reprises et bien plus en profondeur qu'il ne serait possible dans le cadre de cet article. Ce que je propose est d'observer comment certains arguments concernant ces questions furent employés, durant les 
vingt-cinq dernières années, dans le cadre du développement des nombreuses polémiques. Les limites du débat ont été circonscrites de façon très différente à chaque occasion. Pourtant, les discussions ont eu tendance à prendre une forme similaire, pas seulement dans leur référence implicite ou explicite à l'héritage des études historiques germaniques, mais aussi dans leur revendication d'une approche plus traditionnelle du problème d'écrire sur les images.

Une manière simple de présenter ces débats serait d'évaluer depuis le début les conséquences de ce statut à double visage de la discipline. Dans la mesure où l'histoire de l'art est de l'histoire, elle doit inévitablement être considérée comme la branche spécialisée d'une recherche globale qui s'est scindée successivement en domaines d'expertise spécifique tout en conservant son identité par rapport aux disciplines philosophiques et philologiques. En d'autres termes, nous devrions attendre d'elle qu'elle reflète certains développements disciplinaires de l'Histoire, et particulièrement ceux concernant les dimensions culturelle et intellectuelle de celle-ci. Dans le même temps, s'intéressant à des objets d'art spécifiques, l'histoire de l'art ne peut être complètement coupée de l'évolution, particulièrement au long des trois derniers siècles, des langages spécialisés dans la description et l'évaluation des œuvres d'art et de leurs effets, qui peuvent être classés sous l'appellation générique de "critique d'art $\aleph^{22}$. Elle ne peut non plus être totalement détachée de la vision historique que les artistes d'aujourd'hui, et les critiques d'art, considèrent comme appropriée à la compréhension de l'art de leur époque. Comme nous le verrons, la façon dont les débats évoqués ici ont évolué implique de devoir poser à l'approche historique de l'art des questions comme: quels critères déterminer pour le genre d'objets sur lesquels l'historien d'art choisit d'écrire? La catégorie du «visuel » est-elle suffisante en ellemême pour déterminer où l'on doit fixer les limites? Jusqu'à quel point les perceptions de l'état actuel de l'art peuvent-elles être reliées à la vision historique de l'historien d'art? De telles questions, et particulièrement la dernière, pourraient finalement ne jamais trouver de réponses. Mais cela ne signifie pas qu'il faille y renoncer dès le départ.

\section{"Social Art History » et « New art history »}

21 Un point de départ communément admis pour les débats qui ont animé la discipline dans le dernier quart du siècle pourrait être le cri d'alarme lancé par le jeune historien d'art britannique T. J. Clark, publié dans le Times Literary Supplement du 24 mai 1974. Il déclarait que l'histoire de l'art n'était peut-être pas en crise mais «à bout de souffle, dans un état de dissolution raffinée ", qu'elle avait été déchue du droit dont elle avait bénéficié autrefois (en Allemagne en tout cas) de se ranger parmi les disciplines de recherche les plus avancées, et qu'elle avait réduit son domaine d'investigation intellectuelle ${ }^{23}$. Le fait que T. J. Clark présente les travaux pionniers des historiens d'art allemands de la première moitié du siècle en opposition radicale avec la décadence de sa propre époque mérite évidemment d'être signalé. Mais la nouvelle orientation qu'il a personnellement réussi à donner à l'histoire de l'art ne peut être vue, en aucun cas, comme un simple retour aux discours d'A. Riegl, H. Wölfflin, A. Warburg et leurs confrères théoriciens. Ce qu'il proposait était une nouvelle critique fondée sur une notion revisitée et sophistiquées de l'idéologie. Comme il le dit, «l'idéologie est ce qu'est l'image (picture), et ce qu'elle n'est pas ». Pour polir cette élégante formule, il choisissait une terminologie directement empruntée à L'interprétation des rêves de 
Sigmund Freud: "L'idéologie est le contenu du rêve, sans le travail du rêve " ${ }^{24}$. Le modèle que défendait T.J. Clark pour revigorer la création artistique n'était pas le concept rebattu de l'artiste "représentatif » cher aux marxistes démodés, mais le modèle de subjectivité - plus insaisissable et contradictoire en soi - exploré par des écrits tels que ceux de Walter Benjamin sur Baudelaire ou de Jean-Paul Sartre sur Flaubert.

T. J. Clark ne lançait pas son appel à l'action dans le vide. Les deux livres qu'il avait publiés l'année précédant cet article, The Absolute Bourgeois [trad. fr.: Le bourgeois absolu : les artistes et la politique en France de 1848 à 1851, Villeurbanne, 1992] et The Image of the People [trad. fr.: Une image du peuple: Gustave Courbet et la révolution de 1848, Villeurbanne, 1991], portaient respectivement comme sous-titres : « Artists and Politics in France 1848-1851 » et " Gustave Courbet and the 1848 Revolution ». Au moins autant que son article choc, ces travaux donnaient le ton pour un nouvel examen attentif de l'investissement politique et social de l'artiste, qui impliquait également une investigation profonde des sources primaires. Il est certain que cet exemple-plaidoyer de T. J. Clark fit office de précédent, inspira et stimula les jeunes historiens d'art anglais et américains dans la période qui suivit. Il peut paraître difficile aujourd'hui de ne pas voir l'attention qu'il portait à la fin des années 1960 à l'implication des artistes dans des révolutions ratées, à la lumière de l'histoire politique de l'Europe, et de la France en particulier. Des interprétations révisionnistes, fondées sur des sources d'archives, divulguaient une image plutôt différente de l'histoire de l'art et du mécénat en France, au milieu du XIX siècle $^{25}$. Mais la production même de ces nouvelles approches était, littéralement, un hommage rendu à la fertilité de la position de l'historien. Il est certain que T.J.Clark sut garder notre attention en éveil sur des travaux qui révisent et affinent son idée de l'engagement social de l'artiste moderne ${ }^{26}$.

Il demeure cependant une certaine confusion concernant la signification de la nouvelle orientation que Clark, ainsi qu'un bon nombre de chercheurs de même sensibilité, ont réussi à dessiner. Cela est peut-être dû au fait que l'œuvre de T. J. Clark est aujourd'hui spécifiquement identifiée, dans le monde anglophone, à la formulation "social art history » [histoire sociale de l'art]. Lors d'un colloque de 2000 réalisé par le College Art Association (CAA), «Whatever Happened to the Social Art History? " [Qu'est-il donc arrivé à la social art history?], il est rapidement apparu que les positions de ces chercheurs, en tant qu'historiens, ne furent pas toujours bien comprises et que cette confusion tenait en partie à leur affiliation avec ce que l'on pourrait appeler une histoire «dominante $»^{27}$. Un intellectuel, historien, extérieur au domaine de l'histoire de l'art, a avancé que les travaux des « historiens d'art sociaux » semblaient avoir été essentiellement inspirés par l'histoire sociale du milieu du siècle, avec son orientation marxiste associée à des chercheurs britanniques tels qu'E. P. Thompson et Eric Hobsbawm. Cependant, l'historien d'art américain Thomas Crow (interrogé en raison de ses liens personnels avec la " social art history») protestait, car il s'agissait selon lui d'une totale méprise : les historiens dont il se sentait proche et redevable, étaient des Américains portés vers des intérêts culturels généraux, plutôt que spécifiquement politiques ou économiques, comme Natalie Zemon Davis et Carl Schorske ${ }^{28}$.

Cette différence d'interprétation me semble symptomatique des chemins tortueux qu'il faut emprunter pour suivre l'évolution de l'histoire de l'art dans une période récente. Il a déjà été tiré au clair que la "social art history", dans sa version des années 1970, différait fondamentalement de la tradition marxiste incarnée par les spécialistes 
appartenant à la tradition germanique, comme Arnold Hauser, dont la vision de l'artiste en correspondance avec la société reposait sur la logique de la "théorie du reflet ${ }^{29}$. Il faut dire également que les principaux historiens marxistes anglophones cités ci-dessus n'étaient pas spécialement connus pour porter un grand intérêt à l'art. Lorsqu'il leur arrivait d'aborder une œuvre, quelle qu'elle soit, ils avaient tendance à la considérer comme un phénomène superstructurel ${ }^{30}$. Crow avait donc raison d'insister sur le fait que le genre de recherche historique qui l'attirait personnellement était la nouvelle histoire sociale parce qu'elle était plus aventureuse, qu'elle affichait une perspective culturelle et une plus grande réceptivité aux développements récents survenus dans l'historiographie européenne, comme la «Nouvelle histoire» française. On ne pouvait trouver mieux que les noms de N. Z. Davis et C. Schorske, tous deux extrêmement attentifs aux documents figurés comme source pour ce type de recherche.

Toutefois il faut reconnaître qu'au début des années 1970 cette "social art history » en devenir était en train d'être subsumée dans un phénomène plus général et plus diffus que l'on allait bientôt appeler la "New art history». Au sein de ce vaste ensemble, il y avait assez de place pour que des orientations très différentes puissent s'exprimer. Orientations qui étaient en fait plus caractérisées par leur attitude négative commune envers une grande partie de ce qui avait été écrit récemment au nom de l'histoire de l'art que par leur unanimité sur la recherche d'une nouvelle voie à suivre. Les mots new art history résonnèrent comme un slogan très utile pour rallier des conférenciers, présenter des piles de nouveaux livres et convaincre des collaborateurs de rédiger des articles - ce qui ne vise pas pour autant à déprécier la formule. Le fait que des colloques soient tenus et que de nombreux ouvrages et recueils soient publiés sous cette étiquette permet d'évaluer rétrospectivement certains des changements qui étaient en cours.

On s'accorde à dire que l'appellation "New art history" (augmentée d'un point d'interrogation) fut utilisée publiquement pour la première fois lors d'un colloque organisé en 1982 par les éditeurs de Block, revue d'art publiée à la Middlesex Polytechnic, qui sera décrite quelques années plus tard comme "contenant un dynamisme politique plus courant à Nanterre qu'à la National Gallery » ${ }^{31}$. Dès 1986, l'appellation était suffisamment bien acceptée pour que dix-sept collaborateurs répondent à l'appel à contribution sur le sujet désormais dépourvu de son point d'interrogation. En 1994, elle avait acquis sa mythologie propre des deux côtés de l'Atlantique, comme le confirme l'énorme succès de la conférence, tenue cette année-là au Musée d'art contemporain de Montréal sous le titre « Definitions of visual culture: the New Art History - Revisited " [Définitions de la culture visuelle : revoir la New art history]. Aussi sélectifs et non représentatifs que puissent paraître ces événements rétrospectivement, ils ont le mérite d'attirer l'attention sur un certain nombre de courants qui se développaient parallèlement.

L'un d'eux concerne la question, déjà évoquée ici, des relations de l'histoire de l'art avec l'histoire, question qui ne se limite pas uniquement au rapport de la discipline avec son propre passé mais qui concerne également ses liens avec l'évolution à l'œuvre simultanément dans le champ des sciences historiques. Même si cela peut paraître évident, il n'est pas inutile de répéter que la perception de la nécessité d'une « new art history » était intimement liée à la conviction que la discipline n'avait cessé de décliner, après avoir atteint son apogée dans l'immédiat après-guerre. Peu de successeurs paraissaient dignes de reprendre le flambeau des spécialistes allemands. En 1994, 
Thomas Crow ne voyait plus qu'une seule raison à cette déplorable situation : l'échec des chercheurs américains plus âgés à relever le défi lancé par leurs professeurs. "Le plus grand dommage fut causé par les piètres performances d'une génération entière de chercheurs américains, pour la plupart formés par les émigrés européens après la Seconde Guerre mondiale. Se trouvant face à des étudiants manquant d'ambition, ces professeurs ont réduit leurs exigences, consciemment ou non ", écrivait-il ${ }^{32}$. Pour T. Crow, le problème ne venait pas de la génération d'après-guerre faisant fi de la rigueur et de l'intérêt philosophique des historiens d'art émigrés, mais de la perte de "talents réels dans la recherche en histoire", due à un recul généralisé face à l'ampleur des efforts intellectuels à fournir.

Même s'il a pu paraître justifié de présenter l'histoire de l'art en Amérique comme ayant perdu son nerf intellectuel dans le troisième quart du siècle, cela n'était pas applicable à l'ensemble de l'histoire et plus particulièrement à son approche européenne. Pour moi, il semblait clair, lorsque j'ai répondu aux questions des éditeurs de The New Art History en 1986, que « la destinée de la 'New art history' [était] totalement liée à ce qu'on appelait maintenant la 'Nouvelle histoire' [...]. Il ne peut y avoir aucune ' new art history' si ce n'est dans la mesure où elle participe d'une 'nouvelle histoire' ${ }^{33}$. Sous la rubrique "nouvelle histoire ", je plaçais, bien entendu, des membres de l'école française des Annales, comme Fernand Braudel, Georges Duby et Emmanuel Le Roy Ladurie, aux côtés de Natalie Zemon Davis et Keith Thomas. Je me sentais moins préoccupé que T. Crow par la perte des techniques en matière de recherche d'archives, préférant attirer l'attention sur la nécessité pour les historiens d'art « de procéder à une évaluation autocritique des fondements rhétoriques qui sous-tendent le métier d'historien, telle qu'on peut la trouver chez Michel Foucault, Roland Barthes ou Hayden White $»^{34}$.

Pourtant, les intérêts communs qui animaient les collaborateurs de The New Art History en 1986 allaient bien au-delà de ce problème de relations entre l'histoire de l'art et l'histoire, que ce soit sous ses formes "nouvelles » ou traditionnelles. En maintenant scrupuleusement les contributeurs dans l'ignorance des problèmes abordés par les autres, les éditeurs réussirent à obtenir une profusion d'approches éclectiques qui semblent d'autant plus révélatrices rétrospectivement. Les thèmes traités allaient de la promotion de "petits magazines " pour l'étude de l'histoire des mouvements d'avantgarde (Dawn Ades), des réflexions sur les « implications mutuelles de l'histoire de l'art et du métier de conservateur " (Charles Harrison), aux conséquences de la sémiotique (Margaret Iversen), du féminisme (Lynda Nead) et de la critique esthétique (Michael o'Pray) sur l'histoire de l'art, en passant par l'état des programmes proposés aux étudiants (Marcia Pointon). De façon significative, plusieurs contributions mentionnaient de façon récurrente un choix de domaines interdisciplinaires qui étaient alors en cours de formation et allaient rapidement acquérir leur propre dynamisme : parmi eux, le paysage (à la fois dans le sens de la peinture de paysage et de l'histoire des jardins), l'étude de la muséologie ainsi que l'histoire et la théorie de la photographie ${ }^{35}$.

À cet égard, The New Art History reflétait certainement la vitalité des nouveaux centres d'intérêt qui se développaient à la fois à l'intérieur et en marge de la discipline. Ils trouvaient leur expression dans de nouvelles revues stratégiquement planifiées, ainsi que dans les études de certains auteurs. Critical Inquiry a déjà été mentionné comme un périodique important qui allait ouvrir ses pages à l'histoire de l'art. Representations, qui 
fut d'abord publié en 1983 par les presses de l'University of California sous la corédaction des "nouveaux historicistes " Stephen Greenblatt et Svetlana Alpers, allait offrir un pont très utile entre les spécialistes en études littéraires, le "nouvel historicisme », et la "poétique culturelle » associée à l'anthropologue Clifford Geertz. En 1986, j'avais attiré l'attention sur cette nouvelle revue, ainsi que sur deux autres publications récentes, nées en Grande-Bretagne et éditées par le chercheur anglais John Dixon Hunt: Journal of Garden History et Word \& Image ${ }^{36}$. La première s'est montrée exemplaire en faisant le point sur un débat diffus jusqu'alors, ce qui permit d'élever considérablement les exigences en matière de recherches sur le paysage et d'histoire des jardins. La seconde a été également remarquable, pas seulement en raison du flux continu d'excellents articles qu'elle a accueillis, mais aussi pour avoir incité des études sur une large gamme de sujets qui allaient des enluminures médiévales des manuscrits aux études des emblèmes, de la sémiotique et de la cartographie à la publicité et l'histoire de l'illustration des livres. Il est clair que le succès de ces nouvelles revues n'a pas affecté les chances des publications existantes. En fait, elles ont aidé à revigorer les « journaux internes » de la discipline, en particulier Art History, organe de la British Art Historians' Association depuis les années 1970, ainsi que The Art Bulletin, publié par le College Art Association of America.

31 Un bon nombre de ceux qui étaient associés à la "new art history» avaient choisi de marquer leur divergence avec les problématiques de l'après-guerre en proclamant leur ouverture à la théorie. Ce qui ne signifiait pas uniquement chercher à se mettre au niveau des classiques allemands déjà mentionnés ici, mais assimiler le nouveau matériau - à la fois historique et critique - sur les arts visuels, publié en France à partir des années 1960. En 1988, Norman Bryson lançait sa collection aux presses de l'université de Cambridge intitulée "New Art History and Criticism », avec un recueil de traductions de textes d'auteurs français tels que Julia Kristeva, Louis Marin, Roland Barthes et Michel Foucault. En France, aucun de ces auteurs n'aurait été reconnu comme historien d'art, au sens strict, par la profession. N. Bryson tenait cependant à faire remarquer dans son introduction que les analyses, brillantes et ingénieuses, d'œuvres d'art visuel comme celles d'un poète tel qu'Yves Bonnefoy, d'un historien et philosophe des sciences comme Michel Serres, ou bien d'un critique d'art comme JeanClaude Lebensztejn, sans oublier les figures de renommée internationale déjà citées, pouvaient être une source d'inspiration pour les nouveaux historiens d'art du monde anglophone ${ }^{37}$. L'engagement que la collection de Cambridge avait pris envers les nouveaux écrits français s'illustra particulièrement dans la publication d'essais comme, par exemple, un recueil de textes de Jean-Louis Schefer publiés sous le titre Enigmatic body. Essays on the arts (Cambridge, 1995).

32 La stratégie de N. Bryson impliquait une focalisation, nouvelle et fertile, sur la théorie française, qui complétait l'approche germanique en histoire de l'art, mais qui, dans une certaine mesure, mettait à l'épreuve son hégémonie ${ }^{38}$. Ses travaux des années 1980 avaient établi certaines bases de cette nouvelle orientation, avec Word \& Image: French Painting of the Ancien Régime (1981), qui portait son attention sur la critique d'art de Diderot, et Vision and Painting: The Logic of the Gaze (1983), qui cherchait à supplanter le point de vue psychologique adopté par E. H. Gombrich dans L'art et l'illusion, avec une analyse sémiotique et psychanalytique de la représentation fondée sur les théories de Jacques Lacan. Dans le chapitre "The Pictorial Turn » de Picture theory, W. J. T. Mitchell admet que N. Bryson a réussi à « apporter les dernières nouvelles de France et à tirer l'histoire de l'art de son sommeil dogmatique $»^{39}$. Il reconnaît toutefois " qu'une étude 
plus générale " de l'évolution de l'histoire de l'art anglo-américaine allait devoir retrouver "l'œuvre révolutionnaire" de nombreux autres acteurs importants qui furent publiés à partir des années 1960 : Svetlana Alpers, Michael Baxandall, Rosalind Krauss, Ronald Paulson, Leo Steinberg, Tim J. Clark et Michael Fried. Il est difficile de remettre cette liste en question, tant ses membres ont joué un rôle central dans la construction de la New art history, dans son sens le plus large. Il est également impossible de rendre justice ici, même de façon sommaire, à l'étendue des contributions à l'histoire de l'art apportées par chacun d'eux. Cependant, quelquesunes des personnes citées ci-dessus auront droit à une place centrale dans le débat final que je vais résumer, et que l'on peut considérer comme englobant de nombreuses questions déjà soulevées ici.

\section{Culture visuelle vs autonomie de l'art}

33 Dans les différentes étapes que j'ai essayé de retracer jusqu'à présent, les expressions " social art history" et "new art history" ont servi de symptômes du changement de discours entre le milieu des années 1970 et le milieu des années 1980. La première approche n'a pas été supplantée par la seconde; disons plutôt que de nombreux nouveaux ingrédients s'étaient ajoutés à elle ${ }^{40}$. Les différences de génération ont certainement joué leur rôle en exacerbant ces oscillations entre ces approches méthodologiques et celles qui ont suivi. De fait, lors du colloque de 2000 «Whatever Happened to the Social Art History?» déjà mentionné, il était évident que les historiens d'art de la génération intermédiaire étaient venus soulever des questions beaucoup plus incisives concernant les deux décennies précédentes que T. Crow n'était désireux d'y répondre. James Elkins suggérait que les études sur les nouveaux médiums et les arts visuels avaient simplement supplanté l'histoire sociale, et qu'une pluralité de nouvelles autorités avait remplacé la tyrannie monolithique exercée par Marx et ses épigones. Anne Higonnet accusait la "social art history" de rendre les objets fonction des problèmes au lieu des problèmes fonction des objets, et elle reprochait à une certaine lecture du XIX ${ }^{e}$ siècle de transformer un petit groupe d'artistes en héros ${ }^{41}$. Thomas Crow s'opposait à ces attaques et l'accusait de subjectivité, considérant que l'histoire de l'art pratiquée pendant les années 1970 - tout comme aujourd'hui - se justifiait uniquement par sa profondeur intellectuelle et sa qualité inhérente de recherche universitaire

Ces brèves remarques tirées d'une récente table ronde visent à démontrer une seule chose : que les questions qui ont dominé les débats dans l'histoire de l'art tout au long des vingt-cinq dernières années changent inévitablement, en substance et en importance relative, selon la génération de la personne qui les considère. J'ai commencé par signaler un « tournant historiographique » en histoire de l'art comme la preuve que la discipline s'était de plus en plus affermie sur le plan institutionnel. Mais bien sûr, avoir accès aux sources germaniques et fournir des guides méthodologiques aux étudiants n'était pas le garant d'une écriture plus brillante et intellectuellement stimulante. J'ai passé un certain temps à examiner les débats successifs tournant autour de la « social art history » et de la " new art history ». Ils apportent certainement la preuve solide de l'existence d'un vaste mouvement de renouveau, qui s'est attaché à des secteurs et à des méthodes interdisciplinaires nouveaux, ainsi qu'à redonner à la recherche traditionnelle d'archives un peu de son prestige perdu. Mais un problème persiste toujours à l'intérieur du domaine élargi de l'histoire de l'art, que Mitchell 
diagnostiquait en faisant allusion au «tournant visuel » des sciences humaines. Ce point était déjà implicite dans de nombreuses contributions au débat sur la New art history que j'ai résumé, et il peut s'exprimer par une simple question : l'histoire de l'art est-elle destinée à perdre sa propre identité, pour être subsumée dans l'histoire de la «culture visuelle»?

Cette question fut au cœur de tous les débats des années 1990 et, dans une certaine mesure, elle a pris la place des deux débats déjà résumés. On peut la considérer comme la conséquence irréversible de l'évolution des disciplines adjacentes au domaine de l'histoire de l'art, ainsi qu'une caractéristique du contexte élargi évoqué par W.J. T. Mitchell. Prenons par exemple la position actuelle des études filmiques, dont N. Bryson disait en 1988, avec raison, qu'elles avaient pris l'histoire de l'art de vitesse dans leur nouvel approfondissement théorique et semblent aujourd'hui bien plus proches du domaine de l'historien d'art. Les historiens du cinéma ont porté un intérêt considérable au spectacle $\mathrm{du}$ xix ${ }^{\mathrm{e}}$ siècle ainsi qu'aux aspects techniques et institutionnels des panoramas et des dioramas, qui présageaient l'invention de la cinématographie. Cette confluence peut également être observée dans la récente et rapide évolution de l'histoire et de la théorie de la photographie, même si ses premiers progrès ont été hésitants du côté européen de l'Atlantique. Il est bien plus difficile aujourd'hui que dans les années 1970 de cerner l'«art », en particulier dans le contexte social du $\mathrm{XIX}^{\mathrm{e}}$ siècle. On voit paraitre régulièrement des études qui ne traitent pas seulement de la photographie, mais aussi du domaine négligé de la gravure, comme l'un des moteurs puissants pour la circulation et la communication des images, plutôt que de pâles reflets des « beaux-arts » ${ }^{42}$.

Cependant, les arguments en faveur d'une focalisation sur la « culture visuelle » ont été mis en question dans les années 1990, et pas seulement par les historiens d'art traditionnels dont la mission est de s'occuper des «chefs-d'œuvre » de la peinture occidentale. Dans un numéro spécial d'October de l'été 1996, Rosalind Krauss et Hal Foster rassemblaient une quantité d'importantes contributions à ce débat, sous la forme de réponses d'universitaires américains à un questionnaire sur la culture visuelle, et d'une compilation d'articles solidement argumentés ${ }^{43}$. Le premier d'entre eux, de Kurt W. Forster, est consacré à la figure considérée d'une façon générale comme ayant anticipé l'étude de la culture visuelle, Aby Warburg. K. Forster analyse l'intérêt que portait Warburg à l'ethnographie et revient sur ses comparaisons entre certaines caractéristiques de la culture des Indiens d'Arizona et les images votives en cire très répandues dans la Florence de la Renaissance, jusqu'alors négligées par les historiens d'art en raison de leur "vision expurgée de la Renaissance comme une époque de raffinement $\aleph^{44}$. Cela approfondit la perspective et donne une grande portée à la discussion d'aujourd'hui, que W. J. T. Mitchell développe à son tour considérablement dans un article du même numéro. Il est prêt à reconnaître que la "culture visuelle ", telle qu'elle a été préconisée au cours de la décennie précédente, a "apporté une transformation remarquable dans le monde clos et somnolent de l'histoire de l'art académique ». Mais il s'interroge aussi sur le besoin de l'histoire de l'art d'insister sur " une rhétorique d'innovation et de modernisation ", de se mettre en concurrence avec les «disciplines fondées sur les textes, les études des films et de la culture de masse ». Selon lui, «les œuvres visuelles veulent les mêmes droits que le langage, pas être transformées en langage. Elles ne veulent pas être nivelées au rang d"histoire des 
images', ni exaltées dans une 'histoire de l'art', mais être considérées comme des entités complexes, occupant les multiples positions et identités du sujet $»^{45}$.

Ces commentaires ne signifient pas que W.J. T. Mitchell désavoue ses premières remarques sur le «tournant vers le visuel ». Mais ils invitent les historiens d'art à faire un examen de conscience minutieux avant de prétendre maîtriser le vaste domaine de la culture visuelle. Ceux qui ont répondu au questionnaire d'October - plusieurs d'entre eux venant de domaines adjacents, comme par exemple les études filmiques et culturelles - se montrent à la hauteur de la situation. Il faut noter - puisque depuis ses débuts October est une revue dédiée à l'art contemporain ainsi qu'à l'analyse critique du modernisme - l'importance qu'ils accordent à l'émergence d'un phénomène parallèle dans les arts visuels. Dans leur éditorial, Rosalind Krauss et Hal Foster mentionnent l'existence d'une « mutation de l'avant-garde : un étrange croisement entre les mondes de la production artistique, de l'exposition, de l'enseignement et de l'édition $»^{46}$. Hal Foster développe cette perception dans un article éloquent qui plaide, à la fin, en faveur d'un nouveau concept d'«autonomie stratégique de l'art». Cela ne signifie pas uniquement imiter Kant incitant à « arracher les institutions hors de l'Ancien Régime ", ni singer l'attaque moderniste sur "la priorité des textes iconographiques", mais reconnaître le défi particulier posé aux arts par la technologie de l'information, qui construit « des archives sans musées $»^{47}$.

Cela dit, il est important de reconnaître en même temps à quel point la «culture visuelle» est devenue partie intégrante de l'étude de l'histoire de l'art au cours de l'époque récente dont nous débattons ici. Svetlana Alpers affirme, dans sa réponse au questionnaire d'October, que son utilisation de l'expression « culture visuelle » dans The Art of Describing (1983) [trad. fr. : L'art de dépeindre la peinture hollandaise au XVII siècle, Paris, 1990] dérivait du travail historique de M. Baxandall. Elle fait également remarquer, à juste titre, que son utilisation du concept était différente dans son discours sur l'art du XVII e siècle hollandais où, d'après elle, des notions de vision reliées à l'optique, aux instruments comme les microscopes ou la camera obscura, ou encore des techniques comme la cartographie étaient étroitement liées à la pratique de la peinture. Pour reprendre sa formulation: «Je ne m'intéressais pas uniquement aux talents visuels spécifiques à une culture, mais j'affirmais que, à cet endroit et à cette époque, ces talents faisaient autorité ${ }^{48}$. Malgré ces réserves légitimes, il serait difficile de nier l'importance stratégique de la décision de S. Alpers de se concentrer, à ce moment-là, sur l'exemplarité de cette époque et de ces pratiques. De même qu'il est impossible d'exclure la contribution fondamentale de M. Baxandall de toute étude générale sur la constitution de la culture visuelle en domaine d'étude spécifique depuis ces trente dernières années. Qu'il commente l'effet d'un volume dans la Madonna del Parto de Piero della Francesca en se référant à la façon de jauger le contenu d'un tonneau à la Renaissance ou qu'il teste les limites de l'interprétation iconographique en se référant à l'analyse du Baptême du Christ du même artiste, M. Baxandall réaffirme constamment la valeur intellectuelle, ainsi que la spécificité historique, du processus de vision ${ }^{49}$.

Cela peut certes paraître dénaturer légèrement les faits que de distinguer l'élément de « culture visuelle » comme une tension déstabilisante au sein du domaine de l'histoire de l'art, quand la différence d'approche est effectivement si profondément enracinée qu'elle nous conduit jusqu'aux pères fondateurs. Il a été dit plus d'une fois dans un passé récent que l'historien d'art d'aujourd'hui pouvait choisir de suivre E. Panofsky ou 
A. Warburg, et certains des chercheurs les plus innovants de la génération intermédiaire montrent que d'importantes découvertes, récemment, ont puisé leurs sources dans l'une ou l'autre lignée. Cette étude a cherché à souligner, à de nombreux niveaux, que l'histoire de l'art en Grande-Bretagne est à l'intersection de traditions à la fois concurrentes et convergentes, dont aucune ne peut légitimement être considérée comme faisant autorité. L'orientation "culture visuelle» campe la discipline en l'introduisant avec des ruses de renard dans le territoire des sciences sociales, et bien sûr dans l'Histoire tout court. Ce qui n'empêchera pas l'histoire de l'art de continuer à creuser son propre tunnel, tout comme perdurera la question fondamentale, toujours débattue avec passion, de l'« autonomie » de l'art.

\title{
Nicholas Penny et Patricia Rubin | Réactions et réflexions
}

Afin de mieux appréhender la diversité et la richesse actuelle de l'historiographie anglo-saxonne, Perspective a souhaité soumettre l'essai de réflexion et de synthèse de Stephen Bann à la lecture critique de deux spécialistes internationaux de la période moderne, intimement liés à la GrandeBretagne: Nicholas Penny, actuellement conservateur à la National Gallery of Art de Washington et Patricia Rubin, professeur au Courtauld Institute*. [Perspective]

\begin{abstract}
$* * *$
PERSPeCtive. Stephen Bann insiste sur l'esprit de communauté instauré, pour la jeune discipline qu'est l'histoire de l'art, entre la Grande-Bretagne et les États-Unis. Partagez-vous son point de vue, ou considérez-vous l'histoire de l'art en Grande-Bretagne comme aussi, en un certain sens, nationale? Comment vous-même vous situez-vous dans ces échanges transatlantiques?
\end{abstract}

Nicholas Penny. Un certain nombre d'éminents historiens de l'art britanniques ont occupé des postes universitaires en Amérique du Nord ces dernières décennies: T. J. Clark, Michael Baxandall, le regretté John Shearman sont parmi les exemples les plus connus. Le terme "échanges " ne convient peut-être pas vraiment car aucun mouvement n'a eu lieu en sens inverse. Des deux côtés de l'Atlantique, on trouve chez les chercheurs confirmés les mêmes tabous, les mêmes discours moralisateurs, les mêmes modes intellectuelles, mais l'enseignement diffère d'un point de vue majeur : en Amérique du Nord il est essentiellement centré sur l'art moderne américain, et une telle insularité n'a pas cours en Grande-Bretagne.

En même temps, les conservateurs de musées appartiennent à une communauté réellement internationale. Des conservateurs de Saint-Pétersbourg, Berlin et Paris sont des collègues au même sens que ceux de Los Angeles, Chicago, New York et Londres. C'est dû en partie au nombre croissant d'expositions qui font appel à des prêts internationaux. Ceux d'entre nous qui peuvent acheter des œuvres sur le marché international entretiennent aussi des relations étroites avec les marchands d'art de plusieurs villes européennes, tout autant qu'avec ceux de New York. C'est étrange comme peu de chercheurs en histoire de l'art aujourd'hui sont impliqués dans les questions de réévaluation ou de redécouverte d'un artiste - Stephen Bann dans ses travaux sur Delaroche et sur la gravure de reproduction au XIX siècle fait 
office d'exception notable - mais une grande partie des travaux de recherche vraiment novateurs est effectuée par le marché de l'art.

Patricia Rubin. Un éditorial du premier numéro d'Art History, la revue de la British Association of Art Historians, affirmait qu'« il était de la première responsabilité d'Art History d'envisager le domaine dans un cadre plus large, autrement dit que dans l'exploration de nouveaux champs de recherche, aucun matériau, aucun outil, aucune méthode ni aucun langage ne sera exclu ». La revue fut lancée en 1978 et cet appel à la diversité énoncé dans la revue officielle du milieu correspondait à l'expansion prise par la discipline en Grande-Bretagne au début des années 1970. C'était reconnaître ainsi une dimension internationale qui peut-être est due à l'entrée du pays dans la CEE vers la même époque. Parmi les onze premiers membres du bureau de la revue figurait un représentant de l'Amérique du Nord. Aujourd'hui le bureau compte 16 membres, dont 11 qui viennent d'Amérique du Nord ( 9 des USA et 2 du Canada), une proportion qui révèle à mon sens l'importance de l'alliance nordaméricaine dans l'histoire de l'art en Grande-Bretagne aujourd'hui. La présence d'auteurs britanniques et nord-américains dans les plus grandes maisons d'édition de langue anglaise plaide en faveur de l'existence d'une histoire de l'art « anglophone » plus que de deux communautés distinctes.

On ne peut que souscrire au propos de St. Bann quand il dit que «la recherche universitaire "en histoire de l'art a plus ou moins pris» une dimension internationale " et qu'il démontre que c'est là le résultat de dizaines d'années d'« échanges très fertiles ». Pour autant, on peut noter de substantielles différences institutionnelles entre les États-Unis et le Royaume-Uni, qui ont des répercussions sinon sur les modèles qu'adopte la recherche, du moins sur les manières de la pratiquer. Le système éducatif universitaire américain est structuré de façon hétérogène : les universités locales se distinguent de celles qui dépendent des États, certaines écoles sont financées par des fonds publics, mais un grand nombre des universités les plus réputées sont privées. Le système britannique dépend de fonds délivrés par le gouvernement central et est soumis aux standards et aux normes établis du Higher Education Funding Council. Le Council's Research Assessment Exercise a un poids prépondérant dans l'organisation des départements de recherche ainsi que dans le choix des sujets de recherche. Les orientations de l'Arts and Humanities Research Council, autre organisme gouvernemental à délivrer des subventions, imprègne le climat de la recherche en profondeur. Sa caractéristique est d'encourager les projets de recherche collectifs et interdisciplinaires, sur des thématiques larges. Il soutient aussi la recherche dans les musées du pays et promeut activement les liens entre conservation des collections et recherche et enseignement universitaire. Comme le fait remarquer St. Bann, ce lien trouve des racines anciennes dans l'histoire de l'art britannique. Les nouvelles orientations officielles de la politique de l'AHRC aujourd'hui reviennent en fait à reconnaitre l'intensité des échanges qui existent entre les deux milieux, ce qui n'est pas le cas pour l'histoire de l'art américaine.

S'il est justifié et nécessaire de parler d'un système universitaire britannique, cela n'implique pas que l'histoire de l'art pratiquée dans les universités soit monolithique. Le nom des facultés qui relèvent de la University of London témoigne de la diversité de la discipline : outre le «History of Art department » et les facultés d'histoire de l'art de la University College et du Courtauld Institute, on trouve un « department of 
the History of Art, Film and Visual Culture » au Birkbeck College, un « department of Visual Culture " à Goldsmiths, un « department of Art and Archaeology » à la School of Oriental and African Studies.

De mon point de vue, la principale différence entre l'histoire britannique et l'histoire de l'art américaine tient évidemment à leurs échelles respectives. Outre que la communauté universitaire britanique, comme le fait remarquer St. Bann, est plus récente, elle est tout simplement plus réduite, d'où le fait que les orientations prises, les débats, les découvertes et redécouvertes peuvent avoir des répercussions et des impacts éventuellement plus forts.

Ma carrière témoigne de la communauté d'esprit entre les deux pays - je suis née Américaine, j'ai passé un B.A. et un $\mathrm{PhD}$ dans des universités américaines, j'ai fait mon M.A. au Courtauld où j'enseigne l'histoire de l'art de la Renaissance. Des deux côtés de l'Atlantique mon parcours fleure bon l'influence de l'école de Vienne, à travers l'influence de Johannes Wilde au Courtauld et l'enseignement de Konrad Oberhuber à Harvard. Mon expérience professionnelle a été grandement enrichie par la proximité des communautés de l'université et du musée propre au Royaume-Uni, qui permet échange et familiarité tout en restant toujours stimulant.

PERSPECtive. Quelles sont, à votre avis, les caractéristiques de l'histoire de l'art en GrandeBretagne dans les trente dernières années? Voyez-vous d'autres approches, méthodes, ou objets d'études que ceux développés par Stephen Bann?

Nicholas Penny. À mon avis, le peu d'intérêt porté aux travaux de Roberto Longhi (le critique, le polémiste, l'intellectuel et le « connaisseur ») est aussi symptomatique des limites de l'histoire de l'art britannique que l'est l'ignorance dont pâtissent de grands chercheurs allemands comme Riegl. Pour ce qui concerne l'histoire sociale de l'art, je me dis qu'on devrait mentionner l'intérêt pour le contexte physique des œuvres (la hauteur d'accrochage, l'éclairage d'origine, leur disposition architecturale) et pour les usages ou les propos des œuvres, caractéristique de l'enseignement de Johannes Wilde au Courtauld. Cette approche nourrit encore de façon très fructueuse la recherche britannique sur l'art de la Renaissance. Des spéculations sur la soi-disant peur de la prostitution dans les cafés et les égouts de la Seine que l'on discernerait derrière les couleurs chatoyantes des tableaux impressionnistes me paraissent nettement moins éclairantes que la véritable révolution qui a marqué notre compréhension de l'art baroque religieux qu'on peut lire dans la magistrale monographie de Louise Rice sur Saint-Pierre [Louise Rice, The Altars and Altarpieces of New St. Peter's: Outfitting the Basilica, 1621-1666, Cambridge/New York, 1997], ou l'interprétation, totalement nouvelle, d'une forme architecturale comme reflet des pratiques et des conceptions sociales qu'on trouve dans l'étude de Mark Girouard sur les maisons de campagne victoriennes [Mark Girouard, The Victorian Country House, Oxford, 1971, rééd. New Haven, 1979, 1985].

Le connoisseurship (que certains historiens de l'art cités par Bann qualifient de «fétichisme») joue ou devrait jouer un rôle fondamental pour notre discipline. Et l'extraordinaire estime accordée dans le monde entier durant la seconde moitié du $\mathrm{xx}^{\mathrm{e}}$ siècle à un petit groupe de connoisseurs britanniques du dessin des maîtres anciens mérite d'être soulignée. Là encore, cela était dû en partie à Wilde et à son travail au 
British Museum. N'oublions pas non plus l'implication de la science dans l'examen des œuvres. Wilde fut peut-être le premier grand chercheur à utiliser les rayons X (à Vienne dans les années 1930). Il a encouragé ce genre d'études en Grande-Bretagne et la National Gallery de Londres a joué un rôle pionnier en développant des études des matériaux et des techniques des grands maîtres.

Patricia Rubin. Il serait difficile de contester ce que St. Bann relate si magistralement et avec tant de tact sur les orientations de l'histoire de l'art en Grande-Bretagne depuis une trentaine d'années. Il évoque le "cri d'alarme » de T. J. Clark dans son article du Times Literary Supplement de 1974 qui a fait date, faisant judicieusement remarquer qu'il constitue le " point de départ communément admis pour les débats qui ont animé la discipline ». Il peut être utile de resituer ce texte et la forte résonance dont il a bénéficié dans le contexte du développement des études supérieures au Royaume-Uni dans les années 1960 et 1970, avec la création de nouvelles universités et des écoles de sciences exactes. Dans ces dernières en particulier, la perméabilité des disciplines donnait lieu à des débats et des confrontations d'idées à l'intérieur de programmes d'enseignement qui échappaient à des schémas obsolètes.

Mais quand St. Bann parle des traductions des auteurs français dans la collection de Norman Bryson « New Art History and Criticism » aux éditions Cambridge University Press, il sous-estime peut-être l'impact de la pensée française pour la sémiotique et la psychanalyse et le rôle qu'elle a joué dans le tourbillon intellectuel de l'époque. Dans la Grande-Bretagne d'après-guerre, les textes français présentaient l'avantage d'être plus accessibles au public anglophone, pour des raisons de langue, que les textes allemands. Mettant au défi, peut-être, l'« hégémonie » de "l'approche germanique » en histoire de l'art, ils servaient aussi de passerelles pour la pensée allemande. Et comme le signale Bann, ils proposaient également des fondements théoriques solides pour les concepts qui se voulaient une alternative à l'empirisme traditionnel des études historiques britanniques.

Plus plaisant, évidemment, pour un "pays de commerçants », en particulier lorsqu'il était gouverné par une fille de commerçant [NdR: Margaret Thatcher], fut le « tournant vers le matériau » (material turn) que prit l'histoire de l'art dans les années 1980, quand la consommation et la réception des œuvres furent prises comme des domaines d'étude et que la mode rejoignit le style comme sujet digne de considération pour les historiens de l'art. Les material et visual studies ont leur place dans les domaines d'études de l'histoire de l'art en Grande-Bretagne, de la même manière que l'étude de la technique. Celle-ci est à mettre au compte des fines observations de Roger Fry sur la façon dont les peintres, de la Renaissance ou de l'époque moderne, en usaient véritablement avec le matériau de la peinture. La validité des material et visual studies se lit par exemple dans l'un des deux prix accordés cette année par l'Association of Art Historians au mémoire de master Material culture and the construction of ancestry, legacy, and the self in the life of Bernardo Vecchietti (1514-90). Le candidat était un étudiant du Royal College of Art et du programme de master du Victoria and Albert Museum, double affiliation qui a le mérite de rappeler la nature polymorphe de l'histoire de l'art britannique. L'autre lauréat était l'auteur d'un mémoire intitulé Palaces, Pipas and Party Politic: Visual Construction of Civility in Contemporary China, qui met en évidence ce qu'on pourrait appeler la nouvelle géographie de l'histoire de l'art britannique, marquée par la 
préoccupation croissante de faire bouger les frontières de la discipline, au sens propre comme au sens figuré, pour être cohérent avec les exigences de la mondialisation du savoir.

Perspective. Pour vous, quels sont les apports de la "théorie", de "l'histoire de l'histoire de l'art » à la pratique ou la méthode de l'histoire de l'art en Grande-Bretagne ? Considérez-vous que cette prise en compte de la "New Art History » distingue I'histoire de l'art en Grande-Bretagne de I'histoire de l'art telle qu'elle est pratiquée dans d'autres pays d'Europe?

Nicholas Penny. J'ai le sentiment qu'il y a un mauvais côté de la "New Art History ": ses partisans ont adopté une position anti-establishment radicale. Cela dit ce n'est pas un hasard si leurs publications ont correspondu à un moment où l'enseignement de l'histoire de l'art prenait un essor considérable et si elles peuvent passer pour une manière de promouvoir la profession, en fournissant à une nouvelle discipline universitaire ses cultes à mystère, ses textes sacrés, ses mécanismes d'exclusion, ses rites d'initiation et sa flatterie à l'intention des sommités (« comme l'a si bien montré Untel...»). Dans le même temps, l'intérêt porté à la diversité des œuvres s'est notablement amoindri et les étudiants qui travaillent sur le XIX siècle n'ont souvent aucune connaissance de la Renaissance, et les modernistes n'ont en général aucune idée des travaux de recherche sur l'art grec et romain.

Patricia Rubin. Un an après qu'Art History a fait part de son ouverture à des approches et des domaines nouveaux et inexplorés est parue la première livraison de Block. Comme le fait remarquer St. Bann, cette revue était publiée par la Middlesex Polytechnic. Plus un collectif qu'un périodique, son propos était ouvertement politique, ses intentions, critiques et ses orientations, rigoureusement théoriques. Elle entendait substituer au discours sur l'art pour l'art les "problèmes sociaux, économiques et idéologiques » de la culture visuelle.

L'émergence de ces publications qui se répondent représente une partie de l'éventail de l'histoire de l'art à un moment que l'on peut considérer comme sa refondation, moment à la fois œedipien et politique. L'histoire de l'art universitaire en GrandeBretagne est, à mon avis, singulière en ce qu'elle dispose d'une date de naissance bien repérable. Le syntagme "art history», bien qu'étant une traduction de Kunstgeschichte, n'avait pas l'honneur d'être considéré comme wissenschaftlich [scientifique]. Il a fallu attendre la fondation du Courtauld en 1931, sur le modèle du Department of Fine Arts du Fogg Art Museum de Harvard - et cette généalogie dit bien la nature anglo-américaine de l'histoire de l'art britannique. De fait, ce n'est que récemment que les vénérables universités d'Oxford et de Cambridge ont jugé l'histoire de l'art digne de constituer le contenu d'un BA (licence) à part entière phénomène encouragé par le développement de la discipline en dehors d'« Oxbridge » et qui lui confère une indépendance inhabituelle dans les sciences humaines en Grande-Bretagne.

Dans les années 1970, les assauts subis par les histoires de l'art orthodoxes se nourrissaient d'un désir très sain de mettre à bas l'hégémonie de ce qui était devenu une pratique dépourvue de réflexivité, malgré un réel degré d'excellence: une histoire de l'art qui prenait en compte la forme et la fonction, le style et le sujet, mais aussi qui excluait systématiquement les implications idéologiques de la production 
artistique. D'où un espace fécond ouvert à des questionnements sur les prémisses de l'enquête historique. Pour cette raison, il est difficile de parler d'« histoire de l'art en Grande-Bretagne » comme d'une entité unique. Dans les générations précédentes, elle était constituée d'une multiplicité de perspectives - des histoires plutôt qu'une seule histoire de l'art - et d'une interrogation approfondie sur ce qu'implique le fait d'employer le mot «art». Une fois cela admis, ce qui est intéressant est la résistance des deux notions, « art » et « histoire».

Outre ce pluralisme fructueux, l'histoire de l'art en Grande-Bretagne se distingue peut-être de celle des autres pays d'Europe par sa préoccupation constante au contexte, qu'il soit théorique ou historique. Finalement, l'anglais est une langue didactique, d'où le fait que l'expression des idées, quelle que soit la grille interprétative, exige un cadre explicatif.

PERSPECTIVE. Toujours dans ces problèmes de particularismes ou de traditions nationales, y at-il encore un style (ou des styles...) d'écriture de l'histoire de l'art propre à la Grande-Bretagne?

Nicholas Penny. Autrefois, les historiens de l'art britanniques écrivaient sans aucun problème des textes sur l'art accessibles. Certains universitaires, évidemment, préfèrent être abscons. Et la pression carriériste a renforcé un mimétisme en la matière, du coup un nouveau jargon n'a pas mis longtemps à s'installer de part et d'autre de l'Atlantique. Mais il y a quantité de bons textes sur l'art, tout autant américains que britanniques, et quand ils sont bons, en général, on peut facilement identifier la nationalité de leurs auteurs!

Patricia Rubin. S'agissant d'un trait caractéristique de l'histoire de l'art britannique, on peut parler d'un genre plutôt que d'une écriture. St. Bann fait remarquer le caractère littéraire de l'écriture sur l'art avant l'existence d'une l'histoire de l'art britannique (telle qu'on peut la définir avant son institutionnalisation par la fondation du Courtauld). À l'intérieur de ce genre - monographies, catalogues, articles -, on trouve aussi des espèces, ou des types, qui peuvent aussi dépendre du choix du sujet. Écrire sur l'art contemporain suppose la plupart du temps une écriture différente de celle sur les autres périodes, même si toutes ont en commun les questions d'identité politique, les questions matérielles ou esthétiques, pour ne pas en citer plus. Cela dit, l'écriture britannique n'est pas à l'aise avec le mode suggestif et itératif de l'essai, qui semble caractéristique d'une écriture française influente et fort admirée, surtout celle qui est nourrie de la tradition philosophique. Elle supporte également mal la comparaison avec le formalisme rhétorique allusif de la tradition longhienne des textes italiens sur l'art ou la rigueur philologique des travaux des étudiants de l'École normale supérieure de Pise. Une grande part des textes allemands d'histoire de l'art n'est pas encore traduite, d'où la mention fréquente et bienvenue des ouvrages d'Hans Belting, par exemple, alors que les autres auteurs sont relativement passés sous silence. Malgré la présence du Warburg Institute à Londres depuis 1933, ce n'est que tout récemment que les paradoxes et les ambiguïtés fascinants d'Aby Warburg ont commencé à faire leur effet sur l'histoire de l'art britannique - du fait de la traduction en 1999 de son essai The Renewal of Pagan Antiquity grâce à la subvention du Getty Research Institute. Mais, et c'est là, je le répète, la force d'une relative jeunesse et peut-être ce qui reste de la confiance de 
ceux qui furent détenteurs d'un empire, l'histoire de l'art britannique est avide, toujours enthousiaste à l'idée de conquérir de nouveaux territoires intellectuels, et cela ne peut être que fructueux.

\section{NOTES}

1. Heinrich Dilly a calculé que 252 historiens d'art avaient émigré des pays germanophones dans la période précédant la guerre, ce nombre représentant environ le quart de toute la profession. La majeure partie d'entre eux s'est installée en Grande-Bretagne et aux États-Unis.

2. Ce qui inclut, parmi les historiens d'art dont nous parlerons le plus ici, Michael Baxandall, Richard Wollheim, Timothy James Clark, Thomas Crow et Norman Bryson.

3. Voir The Times, 25 juillet 1939. Le Barber Institute ne se présentait pas, cependant, comme un lieu d'enseignement et de recherche en histoire de l'art mais comme « un centre culturel pour Birmingham ». Il n'a commencé à offrir un programme complet d'études d'histoire de l'art au sein de l'université que dans un passé relativement récent.

4. Chaire créée en l'honneur de Felix Joseph Slade (1788-1868), collectionneur, par les universités d'Oxford, Cambridge et Londres [NdR].

5. Pour la carrière de Charles Eastlake, voir David Robertson, Sir Charles Eastlake and the Victorian Art World, Princeton, 1978, où il écrit: «Aucun Anglais de son époque n'avait plus de connaissances en art » (p. 244). D. Robertson étend la comparaison aux étrangers en incluant des conservateurs de musées à Paris et à Berlin; enfin il signale la dette évidente de Bernard Berenson envers Materials for a History of Oil Painting (1847) du même C. Eastlake.

6. Voir en particulier le célèbre essai sur l'école de Giorgione, où Walter Pater met en doute toute cette critique négative du «nouveau Vasari » qui a eu pour effet de réduire le corpus de peintures authentiques de l'artiste. Il réhabilite au contraire tous les artistes «très différents chez qui on peut déceler une influence, un esprit ou un style artistique, et à qui peuvent être réellement attribuées quantité des œuvres présumées de Giorgione »; plus loin dans l'essai, il identifie cet esprit (pour le paysage) avec "ce qu'en Angleterre nous appelons 'park scenery' " (Walter Pater, The Renaissance, Londres, 1925, p. 154 et 159 ; la première version de cet essai a été publiée en 1877 ; traduction française partielle : Essais sur l'art et la Renaissance, Paris, 1985). Quant à New History of Painting in Italy, de Sir Joseph Archer Crowe et Giovanni Battista Cavalcaselle, il fut publié à Londres en 1864 (pour un traitement plus approfondi de l'influence exercée par W. Pater sur ses contemporains européens, voir Stephen Bann éd., The Reception of Walter Pater in Europe, Londres, $2003[\mathrm{NdR}])$.

7. Alex Potts commente la différence entre W. Pater et John A. Symonds en ces termes : «Pour Symonds, la Renaissance s'identifiait à un moment historique concret, lorsque des changements politiques [...] avaient permis à l'humanité de trouver une nouvelle liberté de penser et d'agir [...]. Avec Pater, la question était la formation d'une nouvelle façon d'exister dans le monde et par rapport à lui ». Voir Alex Potts, « Pungent Prophecies of Art: Symonds, Pater and Michelangelo », dans John Pemble éd., John Addington Symonds: Culture and the Demon Desire, Londres, 2000, p. 106.

8. Pour ce qui concerne la réception de l'œuvre d'Adrian Stokes, voir Stephen Bann éd., The Coral Mind: Adrian Stokes' engagement with architecture, art history, criticism and psychoanalysis, University Park (PA), 2007 [NdR]. 
9. Voir le recueil d'essais, très utile, sur les ouvrages de Michael Baxandall dans Art History, 21, 4, 1998, où Allan Langdale discute des différences d'opinion entre Baxandall et Gombrich sur le concept de « l'œil de l'époque ». La contribution de Baxandall à établir le «style cognitif » d'une époque, dont nous parlerons plus loin, diffère nettement de la position résolument antihégélienne de Gombrich.

10. Les lectures faites lors des obsèques de Francis Haskell à la Chapelle du King's College à Cambridge le 10 juin 2000, judicieusement choisies, incluaient le passage dans lequel William Hazlitt raconte sa première visite au Louvre, dans l'essai «On the Pleasure of Painting » (publié dans Table Talk, Essays on Men and Manners, Londres, 1822).

11. Michael Podro, The Critical Historians of Art, Oxford, 1982, p. v.

12. Michael Ann Holly, Panofsky and the Foundations of Art History, Ithaca (NY), 1984. Holly donne le ton de son enquête en posant la question suivante: «Comment, alors, pouvons-nous parler avec assurance de l'histoire de l'art comme d'une 'science', et d'ailleurs, comment pouvons-nous même l'appeler 'histoire', si nous refusons de reconnaître le caractère historique de ses propres principes et techniques?» (cité dans mon compte rendu publié dans History and Theory, 25/2, 1986, p. 200).

13. Alois Riegl, Problems of Style: Foundations for a History of Ornament, Princeton, 1992, p. XXIII.

14. Erwin Panofsky, Perspective as Symbolic Form, New York, 1991, p. 7.

15. Otto Pächt, The Practice of Art History: Reflections on Method, Londres, 1999, p. 13.

16. Voir Paul Duro, Michael Greenhalgh éd., Essential Art History, Londres, 1992 ; Robert S. Nelson, Richard Shiff éd., Critical Terms for Art History, Chicago, 1996 ; Mark A. Cheetham, Michael Ann Holly, Keith Moxey éd., The Subjects of Art History: Historical Objects in Contemporary Perspective, Cambridge, 1998. Je dois signaler en passant la très utile bibliographie dans « Historiography and Methodology of Art History (1972-1994) », dans Nelson, Shiff éd., Critical Terms..., p. XV-XVI.

17. Comme on pouvait s'y attendre, l'Allemagne a été la première dans l'étude de l'évolution de sa propre tradition historique en art. Voir Willibald Sauerländer, «L'Allemagne et la 'Kunstgeschichte' ", dans Revue de l'Art, 45, 1979, p. 4-8, qui résume en détail le matériau de Heinrich Dilly, Kunstgeschichte als Institution, Francfort, 1979. Voir aussi Thomas Gaehtgens, "Étudier l'histoire de l'art en Allemagne ", dans Revue de l'Art, 126, 1999, p. 4-8, pour un état optimiste de la situation actuelle en Allemagne. La France fut lente à suivre, mais la remarquable, et spécifique, évolution de l'histoire de l'art dans le pays a été révélée pour la première fois par Lyne Therrien, L'histoire de l'art en France, Paris, 1998.

18. "If a pictorial turn is indeed occurring in the human sciences, art history could very well find its theoretical marginality transformed into a position of intellectual centrality, in the form of a challenge to offer an account of its principal theoretical object - visual representation - that will be usable by other disciplines in the human sciences. Tending to the masterpieces of Western painting will clearly not be enough. A broad, interdisciplinary critique will be required, one that takes into account parallel efforts such as the long struggle of film studies to come up with an adequate mediation of linguistic and imagistic models for cinema and to situate the film medium in the larger context of visual culture ", W. J. Thomas Mitchell, Picture Theory, Chicago, 1994, p. 15. La contribution de Mitchell à l'histoire de l'art a été spécialement remarquable dans des domaines comme l'ekphrasis et la critique d'art, ainsi que l'histoire sociale du paysage. Sous sa direction éditoriale, le magazine Critical Inquiry, basé à Chicago, surtout consacré à la critique littéraire, est devenu également un lieu de publication pour des essais majeurs sur la recherche historique en art, comme ceux de Michael Fried.

19. Cependant, il est très conscient de décrire une situation qui évolue rapidement: «Il n'y a plus de doute, la discipline de l'histoire de l'art, qui est longtemps restée à la traîne, qui a peutêtre été, de toutes les disciplines des sciences humaines, la plus lente à se développer, et la dernière à être au courant des changements qui se produisaient chez ses voisins les plus proches, 
a maintenant, indubitablement, commencé à se transformer ", dans Norman Bryson, Calligram: Essays in New Art History from France, Cambridge, 1988, p. XIII.

20. Les autres articles du recueil History and Historians in the Twentieth Century dont est issu ce texte de S. Bann sont, outre l'introduction par Peter Burke, «The Middle Ages, or Getting Less Medieval with the Past " par Miri Rubin, «The City» par Peter Clark, «Historians and the Nation » par John Breuilly, «The Orient: British Historical Writing about Asia since 1890 » par Christopher Alan Bayly, "Gender » par Ludmilla Jordanova, «Population History » par Edward Anthony Wrigley, «Disease and the Historian» par Roy Porter, «Class» par David Feldman et « Historiography and Philosophy of History» par Peter Burke [NdR].

21. Voir l'introduction de Christopher Wood à Practice of Art History de Pächt, p. 9-18, et aussi son introduction à Perspective... d'Erwin Panofsky, où est étudiée la relation entre l'iconologie de Panofsky et la méthode philologique, p. 7-24.

22. Il est communément admis que le travail de Johann J. Winckelmann est d'une importance cruciale dans les deux domaines. Voir Alex Potts, Flesh and the Ideal: Winckelmann and the Origins of Art History, Yale, 1994, et idem, "'Sans tête, ni bras, ni jambes'. La description du Torse du Belvédère de Winckelmann », dans Larys Frogier, Jean-Marc Poinsot éd., La Description, Chateaugiron, 1997, p. 19-33.

23. Nelson, Shiff, 1996, cité n. 16, p. XII. Robert Nelson note également l'observation parallèle de Hubert Damisch, en 1975, sur le déclin que l'histoire de l'art a connu après "sa grande époque ", et ajoute qu'elle fut «'totalement incapable de renouveler sa méthode', en référence à des développements théoriques dans d'autres domaines ». L'article de Tim J. Clark est reproduit intégralement dans Eric Fernie éd., Art History and its Methods, Londres, 1995, p. 248-53.

24. Cité dans Fernie éd., 1995, cité n. 23, p. 251.

25. Je me réfère précisément au livre d'une historienne d'art française, Chantal Georgel, 1848 - La République et l'art vivant, Paris, 1998. Elle revendique la poursuite d'une stratégie «inverse » de celle de Tim J. Clark: "Partant de l'hypothèse qu'il devrait y avoir un art spécifique à la République, après avoir étudié les objectifs de l'administration des beaux-arts, celui-ci interroge longuement les œuvres d'artistes susceptibles d'avoir été influencés par les idées nouvelles ou par les événements - Delacroix, Daumier... - afin d'y déceler l'existence d'un 'art républicain' dont il trouve finalement peu de traces. Il y voit un échec [...]. Nous interrogeons au contraire l'ensemble des archives, afin de voir si l'État lui-même, par le biais de son administration artistique, peut être tenu ou non pour responsable d'orientations artistiques nouvelles. Autrement dit, nous ne partons pas ni des artistes ni de leur œuvre contemporaine de la République; nous ne cherchons pas à analyser comment les événements ou l'air du temps' auraient pu ou non influencer leur travail, mais au contraire comment la République elle-même a entendu influer sur le cours de la vie artistique » (p. 9).

26. Pour un bon exposé sur cette notion de médiation sociale, à la lumière de son étude, Farewell to an Idea: Episodes from an History of Modernism, Yale, 1999; voir Gail Day, "Persisting and Mediating: T. J. Clark and the Pain of 'the Unattainable Beyond' ", dans Art History, 23, 1, 2000, p. 1-18.

27. Le colloque s'est tenu lors d'une rencontre à New York présidée par Marc Gotlieb au College Art Association, du 24 au 26 février 2000.

28. Pour l'influence sensible de Carl Schorske sur l'histoire de la culture, voir Michael Roth éd., Rediscovering History: Culture, Politics and the Psyche, Stanford, 1994, volume d'essais publiés en son honneur, auquel Tim J. Clark et Thomas Crow ont contribué. L'intérêt que Natalie Zemon Davis portait à l'image fut attesté en 1982, lorsqu'elle publia ses idées sur le film historique français Le retour de Martin Guerre, auquel elle avait collaboré. Voir Daniel Vigne, Natalie Zemon Davis, JeanClaude Carrière, Le retour de Martin Guerre, Paris, 1982.

29. Les idées personnelles d'Arnold Hauser étaient bien sûr elles-mêmes développées par rapport au sentiment d'un manque d'engagement social de la part des pionniers de la discipline. 
Pour des commentaires pertinents sur la critique de Heinrich Wölfflin par A. Hauser dans sa Philosophy of Art (1963), voir Paul Mattick Jr, «Context », dans Nelson, Shiff éd., 1996, cité n. 16, p. 73-74.

30. Ce qui ne veut pas dire bien sûr que leur travail théorique n'était pas pertinent en tant que cadre pour l'interprétation des œuvres d'art dans leur contexte social et économique. Les commissaires de l'exposition, d'importance majeure, Global Conceptualism: Points of Origin, 1950s-1980s (New York, Queens Museum of Art, décembre 1999-mars 2000) exprimaient clairement leur dette envers Age of Extremes d'Eric Hobsbawm (1994), qui leur avait permis d'établir une périodisation et de retrouver le contexte de la large gamme des œuvres exposées. Pourtant, comme je l'ai signalé dans mon introduction au catalogue, il faut bien admettre que les commentaires de Hobsbawm sur le genre des œuvres exposées sont invariablement négatifs et sans intérêt. Voir le catalogue, du même titre que l'exposition citée, New York, 1999.

31. A. L. Rees, Frances Borzello éd., The New Art History, Londres, 1986, p. 3. L'introduction à ce recueil d'essais fait également bien comprendre la dette du groupe de Block envers l'enseignement dispensé à l'université de Leeds par T. J. Clark pendant la décennie précédente, en particulier son enseignement de MA [master] sur la « social art history».

32. Revoir la New Art History, (colloque, 1994, Montréal), Montréal, 1995, p. 30.

33. Rees, Borzello éd., 1986, cité n. 31, p. 28.

34. Il est intéressant de noter qu'en 1983, Peter Burke concluait son bref essai sur « Rethinking Cultural History » par l'appel à une recrudescence de l'étude de la rhétorique dans son contexte historique, convoquant les historiens de la culture à un engagement dans « la rhétorique de la vie quotidienne ». Cela étant proposé comme antidote à l'attitude " prosaïque » envers l'information historique, parfois illustrée chez les grands classiques de l'histoire de la culture comme Jacob Burckhardt et Johan Huizinga. Voir The Cambridge Review, 104, 2277 (18 novembre 1983), p. 208.

35. Les études de paysage avaient été redynamisées, au début de la décennie, par John Barrell, dans son ouvrage The Dark Side of the Landscape, Cambridge, 1980 ; l'influence de cette excellente analyse par un spécialiste en littérature anglaise s'est reflétée dans le débat public autour de l'exposition sur Richard Wilson par David Solkin à la Tate Gallery (Richard Wilson: The Landscape of Reaction, [cat. expo., Londres, the Tate Gallery, 1982] : exposition commentée par Alex Potts et Neil McWilliam dans "The Landscape of Reaction: Richard Wilson and his Critics", dans Rees, Borzello éd., 1986, cité n. 31, p. 106-119). Les études muséologiques n'étaient pas explicitement le sujet d'une contribution individuelle; à la fin des années 1980, elles avaient généré leur propre élan interdisciplinaire; ce point est mis en évidence dans l'anthologie de Peter Vergo, The New Museology, Londres, 1989. La photographie fut le principal sujet des essais de Victor Burgin et Ian Jeffrey, et elle était également mentionnée, avec le féminisme, par Adrian Rifkin.

36. Rees, Borzello, 1986, cité n. 31, p. 28-29. Journal of Garden History est maintenant connu sous le titre Studies in the History of Gardens and Designed Landscapes. Je dois ajouter que la décennie précédente fut particulièrement marquée par la création de deux revues qui conjuguaient le traitement historique de l'art moderne et contemporain et un niveau exceptionnellement élevé de critique engagée : October, éditée à l'origine à New York par Jeremy Gilbert-Rolfe, Rosalind Krauss et Annette Michaelson, parut pour la première fois au printemps 1976 ; et Macula, éditée à Paris par Yve-Alain Bois, Guy Brett, Jean Clay et Raymonde Hébraud-Carasco, parut dans l'été 1976.

37. Voir Bryson, 1988, cité n. 19. Le seul essai non calqué sur les derniers écrits français sur l'art était celui du sémioticien tchèque Jan Mukarovsky : Art as Semiological Fact, 1934 ; cet article avait toutefois été écrit en français à l'origine, pour être présenté à un congrès international. L'engagement que la collection de Cambridge avait pris envers les nouveaux écrits français s'illustra particulièrement dans la publication d'essais comme, par exemple, le recueil de textes de Jean-Louis Schefer. 
38. Il faut insister sur le fait que l'état fragmentaire de l'histoire de l'art française (avec son manque flagrant d'échanges fertiles comme ceux exposés dans Calligram de N. Bryson), provient en partie d'un développement institutionnel s'étalant sur une longue période, qui impliquait essentiellement des corps indépendants aussi divers que l'École des beaux-arts, l'École du Louvre, le Collège de France et la Sorbonne. Les différents enchaînements en sont minutieusement analysés dans L'histoire de l'art en France de Lyne Therrien, Paris, 1997, chap. 2, 3, 4, 6.

39. W. J. Thomas Mitchell, "The Pictorial Turn», dans Picture theory, Chicago/Londres, 1994, p. 14. Il fait une comparaison intéressante avec Jonathan Culler, qui, dans la décennie précédente, a rendu un "service " similaire à la «critique anglo-américaine». Il est utile de signaler que Culler, dont le Structuralist Poetics fut publié à Londres en 1975, et N. Bryson sont passés d'une université anglaise à une université américaine, pendant que leurs travaux s'acquittaient de ce rôle de transmission.

40. Il est intéressant de noter que, parmi les contributions à l'anthologie de 1986, seul John Tagg a vu qu'il était de son devoir de mettre en doute la mission de la " social art history ". Se référant à T. J. Clark, qui affirmait en 1974 qu'elle était « le lieu où les questions devaient être posées », il énumère diverses critiques des "espérances placées autrefois dans la 'social art history' " (Rees, Borzello éd., 1986, cité n. 31, p. 165-166).

41. T. J. Clark et T. Crow n'ont certainement pas considéré qu'il était de leur devoir de contester le canon des maîtres confirmés, au moins ceux du XIx ${ }^{e}$ siècle. Ce qui signifie que, malgré la grande qualité de l'analyse de peintres majeurs comme David, Courbet et Manet, de grosses lacunes sont apparues lorsqu'il y a eu une tentative d'élargir «l'histoire sociale » au-delà des époques privilégiées. L'étude de Stephen Eisenman avec le contenu revendicateur de son titre, NineteenthCentury Art: A Critical History (Londres, 1994), contient d'excellents passages écrits par T. Crow, mais aussi des arguments usés jusqu'à la corde, comme par exemple dans l'article dédaigneux de l'éditeur « The July Monarchy and the Art of Juste Milieu ».

42. Voir par exemple la remarquable étude d'Anthony Hamber 'A Higher branch of the Art': Photographing the Fine Arts in England 1839-1880, Amsterdam, 1996. L'exposition des œuvres de Paul Delaroche, au Musée des beaux-arts de Nantes puis au Musée Fabre de Montpellier, est probablement la première où un peintre $\mathrm{du} \mathrm{XIX}^{\mathrm{e}}$ siècle est présenté entouré d'un éventail complet de gravures et de photographies associées à son œuvre. Voir Isabelle Julia, Claude CosneauAllemand, Paul Delaroche : Un peintre dans l'histoire, Paris, 1999.

43. Voir October, 77 (été 1996). Parmi ceux qui ont répondu au questionnaire se trouvent plusieurs historiens d'art mentionnés ici : Svetlana Alpers, Thomas Crow, Michael Ann Holly, Keith Moxey et Christopher Wood.

44. Ibid., p. 17.

45. Ibid, p. 82. W. J. T. Mitchell reconnaît qu'il s'insurge contre les affirmations de Norman Bryson, Michael Ann Holly et Keith Moxey dans l'introduction de leur recueil d'essais Visual Culture: Images and Interpretations, Hanovre, 1994.

46. October, 77 (été 1996), p. 4.

47. Ibid., p. 97 et 118-119.

48. Ibid., p. 26.

49. Voir Michael Baxandall, Painting and Experience in Fifteenth-century Italy, Oxford, 1972 (trad. fr. : L'œil du Quattrocento, Paris, 1985) pour la Madonna, et id., Patterns of Intentions : On the Historical Explanation of Pictures, Yale, 1985 (trad. fr. : Les formes de l'intention. Sur l'explication historique des tableaux, Paris, 1991) pour le Baptême.

*. Barthélemy Jobert, contacté pour ce débat, n'a pu malheureusement y participer. 
INDEX

Keywords : historiography, british writing, writing on art, aesthetical critic, image, language, humanities, social sciences, social art history, new art history, visual culture, exchanges

Mots-clés : historiographie, écriture britannique, écriture sur l'art, critique esthétique, image, langage, sciences humaines, culture visuelle, échanges

Index géographique : Grande-Bretagne, États-Unis

Index chronologique : 1900 